\title{
ON THE RANK OF A BINARY FORM
}

\author{
GONZALO COMAS AND MALENA SEIGUER
}

\begin{abstract}
We describe in the space of binary forms of degree $d$ the strata of forms having constant rank. We also give a simple algorithm to determine the rank of a given form.
\end{abstract}

\section{Introduction}

Let $V$ be a vector space of dimension two over an algebraically closed field $K$ of characteristic zero. Let $q$ be a binary form of degree $d$ over $V$. We define the rank of $q$ (denoted $\operatorname{rank} q$ ) to be the least integer $r$ such that $q$ can be written as a sum of $r d$ th powers of linear forms:

$$
q=L_{1}^{d}+\cdots+L_{r}^{d}
$$

In this article we describe the sets of homogeneous forms of a given rank.

We denote with $S_{d, r}$ the set of binary forms of degree $d$ and rank $r$. We show that if a limit of forms in $S_{d, r}$ has rank greater than $r$, then it will have rank greater than or equal to $d-r+2$. More precisely, we prove that

$$
\overline{S_{d, r}} \backslash \overline{S_{d, r-1}}=S_{d, r} \cup S_{d, d-r+2}
$$

for $2 \leq r \leq\left[\frac{d}{2}\right]+1$; and $\overline{S_{d, 1}}=S_{d, 1}$ is the set of $d$ th powers of linear forms (see theorem 21).

We can restate the result of the theorem as follows

$$
\overline{S_{d, r}}=\overline{S_{d, r-1}} \cup S_{d, r} \cup S_{d, d-r+2} .
$$

Applying this result repeatedly, we get a characterization of the possible ranks of degenerated forms

$$
\overline{S_{d, r}}=\bigcup_{i=1}^{r} S_{d, i} \cup \bigcup_{i=d-r+2}^{d} S_{d, i} .
$$

We also show how to determine the rank of a given form $q$ by computing the rank of an explicit matrix, solving a linear system of equations, and deciding if a polynomial constructed from this solution has multiple roots.

Acknowledgments. We wish to thank F. Cukierman for suggesting this problem and for his encouragement. We also thank J.M. Landsberg for useful discussions. 


\section{RANK OF A BINARY FORM}

Let $V=K^{2}$ and let $S_{1}=V^{*}$ be the vector space of linear polynomials in two variables $x, y$. Also let $S_{d}$ be the space of homogeneous polynomials of degree $d$ in variables $x, y$. We define the rank of a binary form $q \in S_{d}$ as follows

Definition 1. The rank of $q$ is the least integer $r$ such that $q$ can be written as

$$
q=L_{1}^{d}+\cdots+L_{r}^{d}
$$

where $L_{i} \in S_{1}, i=1, \ldots, r$.

Let $X \subset \mathbb{P}\left(S_{d}\right)$ be the Veronese curve, that is, the image of the map

$$
\begin{aligned}
\mathbb{P}\left(S_{1}\right) & \rightarrow \mathbb{P}\left(S_{d}\right) \\
{[L] } & \mapsto\left[L^{d}\right] .
\end{aligned}
$$

Then the rank of a binary form $q$ is the least integer $r$ such that $[q]$ lies on a linear space spanned by $r$ elements lying on $X$. For instance, the forms of rank one are exactly those lying on $X$, and those lying on a secant line to $X$ have rank less than or equal to 2 . However, not all forms lying on the secant variety to the curve have rank less than or equal to 2: in fact, a form lying on a tangent line but not on the curve has rank $d$ ( $[\mathrm{Ha}$, pag. 147). Therefore, this notion of rank does not behave upper-semicontinously.

Similarly, forms lying on a secant $k$-plane spanned by $k+1$ points of the curve have rank less than or equal to $k+1$, but their degenerations may have higher rank.

Let

$$
S_{d, r}=\left\{q \in S_{d}: \operatorname{rank} q=r\right\}
$$

be the set of forms of rank $r$. Let $\operatorname{Sec}^{k}(X)$ denote the $(k+1)$-secant variety of $X$, that is, the variety defined as the closure of the union of $k$-planes spanned by $k+1$ points in $X$. Note that $\operatorname{Sec}^{k}(X)$ is the projectivization of the closure of $S_{d, k+1}$, that is, $\operatorname{Sec}^{k}(X)=\left\{[q] \in \mathbb{P}\left(S_{d}\right): q \in \overline{S_{d, k+1}}\right\}$.

We will prove the following theorem

Theorem 2. For each integer $k, 0 \leq k \leq\left[\frac{d}{2}\right]$, we have

$$
\overline{S_{d, k+1}} \backslash \overline{S_{d, k}}=S_{d, k+1} \cup S_{d, d-k+1}
$$

where $\overline{S_{d, 0}}=\emptyset$.

For $q \in \overline{S_{d, k+1}} \backslash \overline{S_{d, k}}$ we have $\operatorname{rank} q=k+1$ if and only if $[q]$ lies on a secant $k$-plane to $X$; otherwise, $\operatorname{rank} q=d-k+1$.

For the proof, we will use the following alternative way of describing the Veronese curve. Consider the map

$$
\begin{array}{ccc}
\mathbb{P}^{1}(K) & \rightarrow & \mathbb{P}\left(S_{d}^{*}\right) \\
{[\alpha]} & \mapsto & {[e v(\alpha)]}
\end{array}
$$


where $e v(\alpha)$ is the linear functional given by evaluation of polynomials at $\alpha \in K^{2}$. The Veronese curve is the image of this map. More precisely, let $\{x, y\}$ be a basis for $S_{1}$, and $\left\{x^{d}, x^{d-1} y, \ldots, x y^{d-1}, y^{d}\right\}$ a basis for $S_{d}$. We consider in $S_{d}^{*}$ the base dual to that of $S_{d}$. Then the image of a point in $\mathbb{P}\left(S_{1}\right)$ with homogeneous coordinates $[t, u]$ by the first map has homogeneous coordinates $\left[t^{d},\left(\begin{array}{l}d \\ 1\end{array}\right) t^{d-1} u, \ldots,\left(\begin{array}{c}d \\ d-1\end{array}\right) t u^{d-1}, u^{d}\right]$ in $\mathbb{P}\left(S_{d}\right)$. On the other hand, a point in $\mathbb{P}^{1}(K)$ with homogeneous coordinates $[t, u]$ is mapped by the second map to a point in $\mathbb{P}\left(S_{d}^{*}\right)$ with homogeneous coordinates $\left[t^{d}, t^{d-1} u, \ldots, t u^{d-1}, u^{d}\right]$.

Therefore, the isomorphism given by

$$
\begin{array}{clc}
\mathbb{P}\left(S_{d}^{*}\right) & \rightarrow \mathbb{P}\left(S_{d}\right) \\
{\left[Z_{0}, Z_{1}, \ldots, Z_{d-1}, Z_{d}\right]} & \mapsto\left[Z_{0},\left(\begin{array}{c}
d \\
1
\end{array}\right) Z_{1}, \ldots,\left(\begin{array}{c}
d \\
d-1
\end{array}\right) Z_{d-1}, Z_{d}\right]
\end{array}
$$

restricts to an isomorphism between the image of the second Veronese map and the first one.

Using this isomorphism, we define the rank of a functional $\varphi \in S_{d}^{*}$ as follows

Definition 3. Let $\varphi \in S_{d}^{*}$ be a linear functional. The rank of $\varphi$ is the least integer $r$ such that $\varphi$ can be written as

$$
\varphi=e v\left(\alpha_{1}\right)+\cdots+e v\left(\alpha_{r}\right)
$$

where $\alpha_{i} \in K^{2}, i=1, \ldots, r$.

Notice that if $\varphi=\lambda_{1} \operatorname{ev}\left(\alpha_{1}\right)+\cdots+\lambda_{r} \operatorname{ev}\left(\alpha_{r}\right)$, then $\varphi$ is the sum of $\operatorname{ev}\left(\alpha_{i}^{\prime}\right)$, where $\alpha_{i}^{\prime}=\omega_{i} \alpha_{i}$ and $\omega_{i}^{d}=\lambda_{i}$.

We will need two results regarding secant varieties to the Veronese curve ( $\mathrm{Ha}$ ), propositions 11.32 and 9.7).

Proposition 4. Let $X \subset \mathbb{P}\left(S_{d}^{*}\right)$ be the Veronese curve. The secant variety $\operatorname{Sec}^{k}(X)$ has dimension $\min \{2 k+1, d\}$.

Note that if $d=2 n$ or $d=2 n+1, \operatorname{Sec}^{n}(X)=\mathbb{P}^{d}$. This means that computing the rank of points lying on $\operatorname{Sec}^{k}(X)$ for $k \leq\left[\frac{d}{2}\right]$ gives a complete stratification by rank.

Given $[\varphi] \in \mathbb{P}\left(S_{d}^{*}\right)$, we will consider its homogeneous coordinates $\left[Z_{0}, Z_{1}, \ldots, Z_{d}\right]$ where $Z_{i}=\varphi\left(x^{d-i} y^{i}\right)$.

Proposition 5. For any $k \leq s, d-s$, the rank $k$ determinantal variety associated to the matrix

$$
M=\left[\begin{array}{cclc}
Z_{0} & Z_{1} & \ldots & Z_{d-s} \\
Z_{1} & Z_{2} & \ldots & Z_{d-s+1} \\
\vdots & \vdots & & \vdots \\
Z_{s} & Z_{s+1} & \ldots & Z_{d}
\end{array}\right]
$$

is the $k$-secant variety $\operatorname{Sec}^{k-1}(X)$ of the Veronese curve $X \subset \mathbb{P}\left(S_{d}^{*}\right)$. 
We will also need Bertini's Theorem ([Ha], pag. 216)

Theorem 6 (Bertini's Theorem). If $X$ is any quasiprojective variety, $f: X \rightarrow \mathbb{P}^{n}$ a regular map, $H \subseteq \mathbb{P}^{n}$ a general hyperplane, and $Y=f^{-1}(H)$, then

$$
Y_{\text {sing }}=X_{\text {sing }} \cap Y \text {. }
$$

In particular, we will use the formulation: the general member of a linear system on $X$ is smooth outside the singular locus of $X$ and the base locus of the linear system.

We will need the following characterization of the secant variety $\operatorname{Sec}^{k}(X)$, that will allow us to recognize which points lie on secant $k$-planes and which are degenerate.

Lemma 7. Let $[\varphi] \in \mathbb{P}\left(S_{d}^{*}\right)$ and let $k$ be an integer, $0 \leq k<\left[\frac{d}{2}\right]$. Then $[\varphi] \in$ $\operatorname{Sec}^{k}(X)$ if and only if $\varphi\left(L_{1} L_{2} \ldots L_{k+1} S_{d-k-1}\right)=0$ for some linear forms $L_{i}, i=$ $1, \ldots, k+1$.

Proof. Consider the bilinear form $B: S_{d-k-1} \times S_{k+1} \rightarrow K$ given by $B(f, g)=\varphi(f g)$. The matrix of $B$ in basis

$$
\left\{x^{d-k-1}, x^{d-k-2} y, \ldots, y^{d-k-1}\right\} \quad\left\{x^{k+1}, x^{k} y, \ldots, y^{k+1}\right\}
$$

is

$$
M=\left[\begin{array}{cccc}
Z_{0} & Z_{1} & \ldots & Z_{k+1} \\
Z_{1} & Z_{2} & \ldots & Z_{k+2} \\
\vdots & \vdots & & \vdots \\
Z_{d-k-1} & Z_{d-k} & \ldots & Z_{d}
\end{array}\right]
$$

where $Z_{i}=\varphi\left(x^{d-i} y^{i}\right)$ denote the coordinates of $[\varphi]$. Using proposition 5 , $[\varphi] \in$ $\operatorname{Sec}^{k}(X)$ if and only if the rank of this matrix is less than or equal to $k+1$. Equivalently, $[\varphi] \in \operatorname{Sec}^{k}(X)$ if and only if there exists a polynomial $g \in S_{k+1}$ such that $B\left(S_{d-k-1}, g\right)=0$. Factoring $g$ as $g=L_{1} \ldots L_{k+1}$ we get our result.

In the following lemma we give a necessary and sufficient condition for the rank of $\varphi$ to be less than or equal to a given integer $r$. We shall denote with $\Delta_{r}$ the discriminant hypersurface in the affine space $S_{r}$, defined as the locus of polynomials with multiple roots in $\mathbb{P}^{1}(K)$. It is a well known fact that $\Delta_{r}$ is an irreducible hypersurface.

Lemma 8. $\varphi=\sum_{i=1}^{r} \lambda_{i} e v\left(\alpha_{i}\right)$, where $\left[\alpha_{i}\right]$ are distinct points in $\mathbb{P}^{1}(K)$ if and only if the set $A=\left\{f \in S_{r}: \varphi(f g)=0 \forall g \in S_{d-r}\right\}$ is not contained in $\Delta_{r}$.

In particular, every $\varphi$ is a linear combination of $d$ elements in $X$, therefore $d$ is an upper bound for the rank of a binary form. 
Proof. Let $\varphi=\sum_{i=1}^{r} \lambda_{i} e v\left(\alpha_{i}\right)$. The polynomial $f$ with roots $\left[\alpha_{1}\right], \ldots,\left[\alpha_{r}\right]$ lies on $A$, therefore $A$ is not contained in $\Delta_{r}$.

For the converse, let us consider a polynomial $f=L_{1} \cdot L_{2} \ldots L_{r} \in A \backslash \Delta_{r}$, where $L_{1}, \ldots, L_{r}$ are linear forms. For each $i=1, \ldots, r$, let $\left[\alpha_{i}\right]=\left[t_{i}, u_{i}\right] \in \mathbb{P}^{1}(K)$ be the zero of $L_{i}$. Since $f \notin \Delta_{r},\left[\alpha_{1}\right], \ldots,\left[\alpha_{r}\right]$ are distinct. We will show that $\varphi=\sum_{i=1}^{r} \lambda_{i} e v\left(\alpha_{i}\right)$ by proving that $\left\{\varphi, e v\left(\alpha_{1}\right), \ldots, e v\left(\alpha_{r}\right)\right\}$ is a linearly dependent set. Consider the $(r+1) \times(d+1)$ matrix having as its rows the coordinates of these functionals in the base dual to $\left\{x^{d}, x^{d-1} y, \ldots, x y^{d-1}, y^{d}\right\}$.

$$
\left[\begin{array}{cccc}
\varphi\left(x^{d}\right) & \varphi\left(x^{d-1} y\right) & \ldots & \varphi\left(y^{d}\right) \\
t_{1}^{d} & t_{1}^{d-1} u_{1} & \ldots & u_{1}^{d} \\
\vdots & \vdots & \ddots & \vdots \\
t_{r}^{d} & t_{r}^{d-1} u_{r} & \ldots & u_{r}^{d}
\end{array}\right]
$$

We claim that this matrix does not have maximal rank. Let us consider the maximal minor obtained by choosing $r+1$ columns in this matrix. Using the linearity of $\varphi$, this minor can be expressed as $\varphi(g)$, where $g \in S_{d}$ is a polynomial having $\left[\alpha_{1}\right], \ldots,\left[\alpha_{r}\right]$ as roots in $\mathbb{P}^{1}(K)$. Thus, $\varphi(g)=0$.

When $r=d, A$ is the kernel of $\varphi$, which is a hyperplane. Since $\Delta_{d}$ is an irreducible hypersurface, $A \nsubseteq \Delta_{d}$, and therefore $\varphi$ is a linear combination of $d$ elements in $X$.

In the next proposition we show a lower bound for the increase of rank when passing to a degenerate position.

Proposition 9. Let $k \leq\left[\frac{d-1}{2}\right]$. If $[\varphi] \in \operatorname{Sec}^{k}(X)$ does not lie on a $k$-plane generated by $k+1$ points in $X$, then $\operatorname{rank} \varphi \geq d-k+1$.

Proof. We know by lemma 7 that $\varphi$ satisfies the condition

$$
\varphi\left(L_{1}^{n_{1}} \ldots L_{r}^{n_{r}} . f\right)=0 \quad \forall f \in S_{d-k-1}
$$

where $L_{1}, \ldots, L_{r}$ are distinct linear forms, and $\sum_{i=1}^{r} n_{i}=k+1$. As $[\varphi]$ does not lie on a secant $k$-plane to $X$, by using lemma 8 we can assume that $n_{1} \geq 2$. Let $\left[\beta_{i}\right] \in \mathbb{P}^{1}(K)$ be the zero of $L_{i}$ for $i=1, \ldots, r$.

Assume that $\varphi$ is a linear combination of $d-k$ elements in $X$,

$$
\varphi=\sum_{j=1}^{d-k} \lambda_{j} e v\left(\alpha_{j}\right)
$$

Since $r \leq k$, and $k \leq\left[\frac{d-1}{2}\right]$, then $r<d-k$. Therefore at least one $\left[\alpha_{j}\right]$ differs from all $\left[\beta_{i}\right]$. We can assume that $\left[\beta_{i}\right]=\left[\alpha_{i}\right]$ for all $i \leq s$, and $\left[\beta_{i}\right] \neq\left[\alpha_{j}\right]$ for all $i, j>s$, where $s \leq r$. Consider for every $j, 1 \leq j \leq d-k$, a linear form $M_{j}$ having $\alpha_{j}$ as its zero, and let $g$ be the following polynomial of degree $d$

$$
g=\left(\prod_{j=1}^{s} M_{j}\right)\left(\prod_{j=s+2}^{d-k} M_{j}\right)\left(\prod_{i=1}^{r} L_{i}^{n_{i}}\right)
$$


We have $\varphi(g)=0$ by equation (11); and $\varphi(g)=\lambda_{s+1} . c$, where $c \neq 0$, by equation (2). Then $\lambda_{s+1}=0$. Similarly, we get $\lambda_{j}=0$ for all $j \geq s+1$.

Therefore $\varphi=\sum_{j=1}^{s} \lambda_{j} e v\left(\beta_{j}\right)$, and so $\varphi\left(L_{1} \ldots L_{s} . f\right)=0$ for all $f \in S_{d-s}$. But this means that $[\varphi]$ lies on an $(s-1)$-plane generated by $s$ points in $X$, which contradicts the fact that $[\varphi]$ does not lie on a secant $k$-plane.

Lemma 10. We consider for each integer $k \leq\left[\frac{d-1}{2}\right]$ the following vector subspace of $S_{d-k}$

$$
A_{k}=\left\{f \in S_{d-k}: \varphi(f g)=0 \forall g \in S_{k}\right\} .
$$

Then

1. $\operatorname{codim}\left(A_{k}\right)=r \leq k$ if and only if $[\varphi] \in \operatorname{Sec}^{r-1}(X)$.

2. If $\operatorname{codim}\left(A_{k}\right)=k+1$ and $A_{k} \subset \Delta_{d-k}$, then $[\varphi] \in \operatorname{Sec}^{k}(X) \backslash \operatorname{Sec}^{k-1}(X)$ and does not lie on a secant $k$-plane.

Proof. The first statement follows from lemma ․

Let $A=A_{k}$. For the second statement, we use Bertini's Theorem to show that $A$ has base points, since all polynomials in $A$ have singular points. Moreover, one of these base points is a multiple root for every polynomial in $A$.

Let $L_{1}, L_{2}, \ldots, L_{r}$ be linear forms having as zeros the base points of $A$. We have $A=L_{1}^{n_{1}} L_{2}^{n_{2}} \ldots L_{r}^{n_{r}} Z$, where $\sum_{i=1}^{r} n_{i}=m \leq k+1$ and $Z \subseteq S_{d-k-m}$ is a linear subspace of dimension $d-2 k$ having no base points. We claim that $m=k+1$. If $m<k+1$, then $Z$ is a proper subspace of $S_{d-k-m}$ having no base points. Therefore, $\operatorname{dim} S_{1} . Z \geq \operatorname{dim} Z+2$ ([Ha], lemma 9.8 pag. 103), and the codimension of $S_{1} . Z$ in $S_{d-k-m+2}$ is less than or equal to $k-m<k-m+1=\operatorname{codim}(Z)$. If $S_{1} . Z$ is a proper subspace, we can use the same result since $S_{1} . Z$ will have no base points. Repeating this procedure at most $k-m+1$ times, we get a space of codimension 0 , therefore $S_{k-m+1} \cdot Z=S_{d-2 m+1}$. We conclude that $S_{k-m+1} \cdot A=L_{1}^{n_{1}} L_{2}^{n_{2}} \ldots L_{r}^{n_{r}} S_{k-m+1} Z=$ $L_{1}^{n_{1}} L_{2}^{n_{2}} \ldots L_{r}^{n_{r}} S_{d-2 m+1}$. Then

$$
0=\varphi\left(S_{k-m+1} \cdot A \cdot S_{m-1}\right)=\varphi\left(L_{1}^{n_{1}} \ldots L_{r}^{n_{r}} S_{d-2 m+1} S_{m-1}\right)=\varphi\left(L_{1}^{n_{1}} \ldots L_{r}^{n_{r}} S_{d-m}\right) .
$$

This would mean that $[\varphi] \in \operatorname{Sec}^{m-1}(X)$, which contradicts the fact that $\operatorname{codim}(A)=$ $k+1$.

Therefore $k+1=m, A=L_{1}^{n_{1}} \ldots L_{r}^{n_{r}} S_{d-k-1}$, and $[\varphi] \in \operatorname{Sec}^{k}(X)$. Since $A \subset \Delta_{d-k}$, we know by lemma 8 that $[\varphi]$ does not lie on a secant $(d-k-1)$-plane. Then $[\varphi]$ does not lie on a secant $k$-plane, because $k \leq d-k-1$.

The next proposition is reciprocal to proposition 9 .

Proposition 11. Let $k \leq\left[\frac{d-1}{2}\right]$, and let $\varphi$ be a form having rank greater or equal to $d-k+1,[\varphi] \notin \operatorname{Sec}^{k-1}(X)$. Then $[\varphi] \in \operatorname{Sec}^{k}(X) \backslash \operatorname{Sec}^{k-1}(X)$ and $[\varphi]$ does not lie on a secant $k$-plane. 
Proof. It follows directly from lemmas 8 and 10 .

Now we can prove theorem 2 .

Proof of theorem . We rewrite the statement in theorem 2 using secant varieties:

$$
\operatorname{Sec}^{k}(X) \backslash \operatorname{Sec}^{k-1}(X)=\{[\varphi]: \operatorname{rank} \varphi=k+1\} \cup\{[\varphi]: \operatorname{rank} \varphi=d-k+1\}
$$

Note that this implies

$$
\operatorname{Sec}^{k}(X)=\{[\varphi]: \operatorname{rank} \varphi \leq k+1\} \cup\{[\varphi]: \operatorname{rank} \varphi \geq d-k+1\}
$$

for each $k$.

We will use induction on $k$.

For $k=0, \operatorname{Sec}^{0}(X)=X=\{[\varphi]: \operatorname{rank} \varphi=1\} \cup \emptyset$.

For $k>0$, let $[\varphi] \in \operatorname{Sec}^{k}(X) \backslash \operatorname{Sec}^{k-1}(X)$. If $[\varphi]$ lies on a secant $k$-plane, its rank is by definition $k+1$. Otherwise, we have $\operatorname{rank} \varphi \geq d-k+1$ by proposition 9 . If $\operatorname{rank} \varphi>d-k+1,[\varphi]$ lies on $\operatorname{Sec}^{k-1}(X)$ by induction, which contradicts the fact that $[\varphi] \in \operatorname{Sec}^{k}(X) \backslash \operatorname{Sec}^{k-1}(X)$. Therefore $\operatorname{rank}(\varphi)=d-k+1$.

We have proved that

$$
\operatorname{Sec}^{k}(X) \backslash \operatorname{Sec}^{k-1}(X) \subseteq\{[\varphi]: \operatorname{rank} \varphi=k+1\} \cup\{[\varphi]: \operatorname{rank} \varphi=d-k+1\} .
$$

If $\operatorname{rank} \varphi=k+1$, then, by definition, $[\varphi] \in \operatorname{Sec}^{k}(X)$; and $[\varphi] \notin \operatorname{Sec}^{k-1}(X)$ by induction. Finally, if $\operatorname{rank}(\varphi)=d-k+1$ then $[\varphi] \notin \operatorname{Sec}^{k-1}(X)$ by induction and $[\varphi] \in \operatorname{Sec}^{k}(X)$ by proposition 11 .

In the case $d=2 n+1$, this procedure ends when $k=n$, and we have

$$
\operatorname{Sec}^{n}(X) \backslash \operatorname{Sec}^{n-1}(X)=\{[\varphi]: \operatorname{rank} \varphi=n+1\} \cup\{[\varphi]: \operatorname{rank} \varphi=n+2\} .
$$

In the case $d=2 n$ and $k=n$ we cannot use proposition 11. However, we know that $\operatorname{Sec}^{n-1}(X)=\{[\varphi]: \operatorname{rank} \varphi \leq n\} \cup\{[\varphi]: \operatorname{rank} \varphi \geq n+2\}$, and then every form

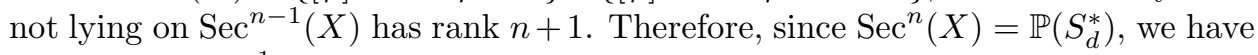
$\operatorname{Sec}^{n}(X) \backslash \operatorname{Sec}^{n-1}(X)=\{[\varphi]: \operatorname{rank} \varphi=n+1\}$.

\section{Computing the RANK}

Next we show how to determine the rank of a binary form $q \in S_{d}$. Firstly we find the integer $k$ such that $q \in \overline{S_{d, k+1}} \backslash \overline{S_{d, d-k+1}}$. Indeed, if $q=Z_{0} x^{d}+\left(\begin{array}{l}d \\ 1\end{array}\right) Z_{1} x^{d-1} y+$ 
$\cdots+\left(\begin{array}{c}d \\ d-1\end{array}\right) Z_{d-1} x y^{d-1}+Z_{d} y^{d}$ then $k+1$ is the rank of one of these two matrices

$$
\left[\begin{array}{cccc}
Z_{0} & Z_{1} & \ldots & Z_{n} \\
Z_{1} & Z_{2} & \ldots & Z_{n+1} \\
\vdots & \vdots & & \vdots \\
Z_{n} & Z_{n+1} & \ldots & Z_{d}
\end{array}\right] \quad \text { or } \quad\left[\begin{array}{cccc}
Z_{0} & Z_{1} & \ldots & Z_{n} \\
Z_{1} & Z_{2} & \ldots & Z_{n+1} \\
\vdots & \vdots & & \vdots \\
Z_{n+1} & Z_{n+2} & \ldots & Z_{d}
\end{array}\right]
$$

depending on wether $d=2 n$ or $d=2 n+1$ (by proposition 5).

Secondly, we decide if $\operatorname{rank} q=k+1$ or $\operatorname{rank} q=d-k+1$. If $d=2 n$, and $k=n$ then $k+1=d-k+1=\operatorname{rank} q$. Otherwise, let

$$
M=\left[\begin{array}{cccc}
Z_{0} & Z_{1} & \ldots & Z_{k+1} \\
Z_{1} & Z_{2} & \ldots & Z_{k+2} \\
\vdots & \vdots & & \vdots \\
Z_{d-k-1} & Z_{d-k} & \ldots & Z_{d}
\end{array}\right]
$$

be the matrix of the bilinear form $B: S_{d-k+1} \times S_{k+1} \rightarrow K$ defined in the proof of proposition 7 . If $\varphi$ denotes the linear functional dual to $q$, then $B$ is the bilinear form associated to $\varphi$. The rank of this matrix is also $k+1$ (by proposition 5), so there is a polynomial $f \in S_{k+1}$, unique up to scalars, such that $B\left(S_{d-k+1}, f\right)=0$.

In order to find $f$, we solve the system

$$
M \cdot\left(\begin{array}{c}
f_{0} \\
f_{1} \\
\vdots \\
f_{k} \\
f_{k+1}
\end{array}\right)=0
$$

and set $f=f_{0} x^{k+1}+f_{1} x^{k} y+\cdots+f_{k} x y^{k}+f_{k+1} y^{k+1}$.

If $f$ has no multiple roots, then $\operatorname{rank} q=k+1$, otherwise $\operatorname{rank} q=d-k+1$. Indeed, if $f$ has $k+1$ distinct roots $\left[t_{1}, u_{1}\right], \ldots,\left[t_{k+1}, u_{k+1}\right] \in \mathbb{P}^{1}(K)$, then $q$ is a linear combination of the $d$ th powers of the linear forms $L_{1}=t_{1} x+u_{1} y, \ldots, L_{k+1}=$ $t_{k+1} x+u_{k+1} y$.

\section{REFERENCES}

[Ha] Harris J., Algebraic Geometry, Graduate Texts in Mathematics, Springer Verlag, Corrected third prining 1995.

Departamento de Matematica FCEyn-UBA, Ciudad Universitaria (1428) Capital FedeRal, Argentina

E-mail address: gcomas@dm.uba.ar

E-mail address: mseiguer@dm.uba.ar 